\title{
Statistical Monitoring of Nonlinear Product and Process Quality Profiles
}

\author{
James D. Williams \\ General Electric Global Research, Niskayuna, NY 12309
}

William H. Woodall and Jeffrey B. Birch

Virginia Polytechnic Institute \& State University, Blacksburg, VA 24061-0439

\begin{abstract}
In many quality control applications, use of a single (or several distinct) quality characteristic(s) is insufficient to characterize the quality of a produced item. In an increasing number of cases, a response curve (profile), is required. Such profiles can frequently be modeled using linear or nonlinear regression models. In recent research others have developed multivariate $T^{2}$ control charts and other methods for monitoring the coefficients in a simple linear regression model of a profile. However, little work has been done to address the monitoring of profiles that can be represented by a parametric nonlinear regression model. Here we extend the use of the $T^{2}$ control chart to monitor the coefficients resulting from a parametric nonlinear regression model fit to profile data. We give three general approaches to the formulation of the $T^{2}$ statistics and determination of the associated upper control limits for Phase I applications. We also consider the use of nonparametric regression methods and the use of metrics to measure deviations from a baseline profile. These approaches are illustrated using the vertical board density profile data presented in Walker and Wright[1].
\end{abstract}

KEY WORDS: Multivariate statistical process control; $T^{2}$-chart; Vertical density profile; Nonlinear regression; Functional data; Minimum volume ellipsoid. 


\section{INTRODUCTION}

In statistical process control (SPC) applications, manufactured items are sampled over time and quality characteristics are measured. Often a product's quality can be determined through measuring several characteristics at each sampling interval. Multivariate $T^{2}$ control charts and other methods have been developed for this scenario. See, for example, Fuchs and Kenett [2] and Mason and Young [3]. Increasingly, however, a sequence of measurements of one or more quality characteristics are taken across some continuum producing a curve or surface that represents the quality of the item. This curve or surface is referred to as a profile. Woodall, Spitzner, Montgomery, and Gupta [4] give an introductory overview of the emerging field of profile monitoring.

Profile data consist of a set of measurements with a response variable $y$ and one or more explanatory variables $x_{j}, j=1, \ldots, k$, which are used to assess the quality of a manufactured item. For example, the density profile of a particleboard is measured on a vertical cross-section, which reveals patterns in board density across the depth of the board. Another example is the estimated dose-response curve of a manufactured drug. Once a batch of the drug is produced, several different doses of the drug are administered to subjects and the responses measured. The resultant dose-response curve summarizes the quality of the particular batch of the drug, indicating the maximal effective response, minimal effective response, and the rate in which the response changes between the two (see Williams, Birch, Woodall, and Ferry [5]). In these examples, a single measurement is insufficient to adequately assess quality. Instead, a relationship between two variables, referred to as the profile, should be monitored over time. Profile data is multivariate, but it is not appropriate to apply standard multivariate control chart methods since this leads to overparameterization. It is more efficient to model the structure of the data via regression techniques. 
In Phase I analysis, we are concerned with distinguishing between in-control conditions and the presence of assignable causes so that in-control parameters may be estimated for further product or process monitoring in Phase II analysis. If out-ofcontrol observations are included in the estimation of in-control parameters, then the subsequent monitoring procedure will be less effective. Therefore it is imperative in Phase I that abnormal profiles be identified and excluded from further analysis. Further, we seek to identify step or ramp shifts (if any) in the mean profile, so that in-control parameters may be estimated to reflect what would be expected from a stable process.

Profiles can take on several different functional forms, depending on the specific application. For many calibration problems, the profile can be represented by a simple linear regression model (see, e.g., Mahmoud and Woodall [6] and Gupta, Montgomery, and Woodall [7]). Kang and Albin [8] proposed two methods, including a multivariate $T^{2}$ control chart, to monitor such profiles. Specifically, we let the subscript $i$ index each individual profile $(i=1, \ldots, m)$ in the historical Phase I data. In the simple linear regression case, the $i^{\text {th }}$ profile is modeled as

$$
y_{i j}=\beta_{i 0}+\beta_{i 1} x_{i j}+\epsilon_{i j}
$$

where $y_{i j}$ is the $j^{\text {th }}$ measurement $(j=1, \ldots, n), \epsilon_{i j}$ is the $j^{t h}$ random error, and $x_{i j}$ is the $j^{\text {th }}$ value of the explanatory variable corresponding to the $i^{\text {th }}$ profile. It is assumed that the values of $x_{i j}$ are the same for all $i$. This assumption is often reasonable since in many engineering applications product or process profiles are measured at fixed values of the explanatory variable at each sampling stage. Kang and Albin's [8] multivariate $T^{2}$ chart is used to monitor simultaneously the $\beta_{0}$, the $y$-intercept, and $\beta_{1}$, the slope. Kim, Mahmoud, and Woodall [9] proposed an alternative approach with better statistical properties such that individual control charts can be used for the $y$-intercept and slope independently. 
In general we refer to any profile that can be modeled by the linear regression function

$$
y_{i j}=\beta_{i 0}+\beta_{i 1} x_{i j 1}+\beta_{i 2} x_{i j 2}+\cdots+\beta_{i k} x_{i j k}+\epsilon_{i j}
$$

as a linear profile, where $x_{i j l}, l=1, \ldots, k$, are $k$ predictor variables. The predictor variables can be the original variables themselves or any function of the variables. In matrix notation, we let $\mathbf{y}_{i}=\left[y_{i 1}, y_{i 2}, \ldots, y_{i n}\right]^{\prime}$ be the vector of responses for profile $i, \boldsymbol{\beta}_{i}=\left[\beta_{i 0}, \beta_{i 1}, \ldots, \beta_{i k}\right]^{\prime}$ be the vector of parameters to be monitored, $\mathbf{x}_{i j}^{\prime}=\left[1, x_{i j 1}, x_{i j 2}, \ldots, x_{i j k}\right]$ be the vector of explanatory variables for item $i$, and $\boldsymbol{\epsilon}_{i}=\left[\epsilon_{i 1}, \epsilon_{i 2}, \ldots, \epsilon_{i n}\right]^{\prime}$ be the corresponding vector of random errors. After collecting the $\mathbf{x}_{i j}^{\prime}$ vectors into an $n \times p$ matrix, where $p=k+1$, as

$$
\mathbf{X}_{i}=\left[\begin{array}{c}
\mathbf{x}_{i 1}^{\prime} \\
\mathbf{x}_{i 2}^{\prime} \\
\vdots \\
\mathbf{x}_{i n}^{\prime}
\end{array}\right]
$$

model (1) can be written in matrix form as

$$
\mathbf{y}_{i}=\mathbf{X}_{i} \boldsymbol{\beta}_{i}+\boldsymbol{\epsilon}_{i}, i=1, \ldots, m
$$

We assume that $\mathbf{X}_{i}$ is the same for each profile and that the vectors $\boldsymbol{\epsilon}_{i}$ are independent and identically distributed (i.i.d.) multivariate normal random vectors with mean vector zero and covariance matrix $\sigma^{2} \mathbf{I}$. For an example of profile monitoring where the covariance matrix is allowed to take on a more general form, see Williams, et al. $[5]$.

Jensen, Hui, and Ghare [10] proposed a control chart based on the F-distribution to monitor the $k+1$ parameters (coefficients) from a multiple linear regression model for Phase II applications. Given the parameter vector estimator for item $i, \hat{\boldsymbol{\beta}}_{i}$, and the target parameter vector $\boldsymbol{\beta}_{0}$, one plots on their control chart the well-known $F$ 
statistic

$$
F_{i}=\left(\hat{\boldsymbol{\beta}}_{i}-\boldsymbol{\beta}_{0}\right)^{\prime} \mathbf{X}_{i}^{\prime} \mathbf{X}_{i}\left(\hat{\boldsymbol{\beta}}_{i}-\boldsymbol{\beta}_{0}\right) /(k+1) s_{i}^{2}
$$

against $i$, where $s_{i}^{2}=\sum_{i=1}^{n}\left(y_{i}-\hat{y}\right)^{2} /(n-p)$. A Phase I procedure for this general linear case has yet to be developed.

In many cases, however, profiles cannot be well-modeled by a linear regression function. Walker and Wright [1] proposed a nonparametric approach for comparing profiles using additive models. Such models do not have a specific functional form and have no model parameters to estimate, but rather one employs smoothing techniques such as kernal smoothing or spline smoothing to model a profile. Nonparametric regression techniques provide great flexibility in modeling the response. One disadvantage of nonparametric smoothing methods is that the subject-specific interpretation of the estimated nonparametric curve may be more difficult, and may not lead the user to discover as easily assignable causes that lead to an out-of-control signal. Ding, Zeng, and Zhou [11] proposed an alternative nonlinear profile monitoring method based on a two stage process of (1) data reduction from a high-dimensional space to a lower-dimensional subspace and (2) employing the control charts methods described in Sullivan [12].

Often, however, scientific theory or subject-matter knowledge leads to a natural nonlinear function that well-describes the profiles. Hence, an alternative method is to model each profile by a nonlinear regression function. A nonlinear profile of an item can be modeled by the nonlinear regression model given generally by

$$
y_{i j}=f\left(\mathbf{x}_{i j}, \boldsymbol{\beta}_{i}\right)+\epsilon_{i j}
$$

where $\mathbf{x}_{i j}$ is a $k \times 1$ vector of regressors for the $j^{\text {th }}$ observation of the $i^{\text {th }}$ profile, $\epsilon_{i j}$ is the random error, $\boldsymbol{\beta}_{i}$ is a $p \times 1$ vector of parameters for profile $i$, and $f$ is nonlinear in the parameters. The random errors $\epsilon_{i j}$ are assumed to be i.i.d. normal random variates with mean zero and variance $\sigma^{2}$. In many applications, there is only 
one regressor $(k=1)$, but there are multiple parameters to monitor $(p>1)$. An example of this form of the model is the 4-parameter logistic model, often used to model dose-response profiles of a drug, given by

$$
y_{i j}=A_{i}+\frac{D_{i}-A_{i}}{1+\left(\frac{x_{i j}}{C_{i}}\right)^{B_{i}}}+\epsilon_{i j}
$$

where $y_{i j}$ is the measured response of the subject exposed to dose $x_{i j}$ for batch $i$, $i=1, \ldots, m, j=1, \ldots, n$. In Equation (3), we have $k=1$ and $p=4$, giving four parameters to monitor, each parameter having a specific interpretation. For example, $A_{i}$ is the upper asymptote parameter, $D_{i}$ is the lower asymptote parameter, $C_{i}$ is the $E D_{50}$ parameter (the dose required to elicit a $50 \%$ response), and $B_{i}$ is the rate parameter for the $i^{\text {th }}$ batch. Another example is the "bathtub" function described in Section 3 where the density of particleboard is measured across the vertical profile. Note that for any given application, the specific form of the nonlinear function, $f$, in Equation (2) must be specified by the user.

In Section 2 of this paper we give a brief review of nonlinear regression. We introduce the multivariate $T^{2}$ statistic in the context of monitoring nonlinear profiles. We then introduce three formulations of the $T^{2}$ statistic and discuss the determination of the upper control limits for the corresponding charts. In addition, a control chart to monitor the variance $\sigma^{2}$ in the context of monitoring profile data is proposed. Finally, we discuss a nonparametric regression approach to monitoring the profiles. In Section 3 we illustrate the $T^{2}$ control charts and the nonparametric approaches using the vertical density profile data of Walker and Wright [1]. In Section 4 we discuss the effects that autocorrelation in the error terms may have on the analysis. Finally, in Section 5 we discuss potential alternative methods and give directions for future research topics in nonlinear profile monitoring. 


\section{METHODOLOGY}

We begin a Phase I analysis with a baseline dataset consisting of $m$ items sampled over time. For each item $i$ we observe a response $y_{i j}$ and a set of predictor variables $\mathbf{x}_{i j}, i=1, \ldots, m, j=1, \ldots, n$, resulting in the quality profile for item $i$, i.e., $\left(y_{i 1}, \mathbf{x}_{i 1}\right),\left(y_{i 2}, \mathbf{x}_{i 2}\right), \ldots,\left(y_{i n}, \mathbf{x}_{i n}\right)$. In this section we develop the methodology to analyze the profiles to gain understanding of the product or process in a Phase I setting.

\subsection{Nonlinear Model Estimation}

For simplicity of notation, we write the scalar model given in Equation (2) in matrix form by stacking the $n$ observations within each profile as $\mathbf{y}_{i}=\left(y_{i 1}, y_{i 2}, \ldots, y_{i n}\right)^{\prime}$, $\mathbf{f}\left(\mathbf{X}_{i}, \boldsymbol{\beta}_{i}\right)=\left(f\left(\mathbf{x}_{i 1}, \boldsymbol{\beta}_{i}\right), f\left(\mathbf{x}_{i 2}, \boldsymbol{\beta}_{i}\right), \ldots, f\left(\mathbf{x}_{i n}, \boldsymbol{\beta}_{i}\right)\right)^{\prime}$, and $\boldsymbol{\epsilon}_{i}=\left(\epsilon_{i 1}, \epsilon_{i 2}, \ldots, \epsilon_{i n}\right)^{\prime}$. The vector form is then given by

$$
\mathbf{y}_{i}=\mathbf{f}\left(\mathbf{X}_{i}, \boldsymbol{\beta}_{i}\right)+\boldsymbol{\epsilon}_{i}, i=1, \ldots, m
$$

For the nonlinear regression model given in Equation (4), estimates of $\boldsymbol{\beta}_{i}$ for each sample must be obtained. This is usually accomplished by employing the GaussNewton procedure and iterating until convergence to obtain the maximum likelihood estimates. Upon convergence of the algorithm, the estimated covariance matrix of $\hat{\boldsymbol{\beta}}_{i}$ is the estimated Fisher information matrix. See Myers [13] or Schabenberger and Pierce [14] for a concise discussion of nonlinear regression model estimation. A more detailed treatment can be found in Gallant [15] and Seber and Wild [16].

Unlike linear regression, the small-sample distribution of parameter estimators in nonlinear regression is unobtainable, even if the errors $\epsilon_{i j}$ are assumed to be i.i.d. normal random variables. Instead, asymptotic results must be applied. Seber and Wild [16] give the asymptotic distribution of $\hat{\boldsymbol{\beta}}_{i}$ and the necessary assumptions and regularity conditions for the asymptotic distribution to be obtained. 


\subsection{Multivariate $T^{2}$ Control Chart}

In order to develop the methodology to monitor nonlinear profiles, we first consider the general framework of the multivariate $T^{2}$ statistic. Given a sample of $m$ independent observation vectors to be monitored, $\mathbf{w}_{i}(i=1, \ldots, m)$, each of dimension $p$, the general form of the $T^{2}$ statistic in Phase I for observation $i$ is

$$
T_{i}^{2}=\left(\mathbf{w}_{i}-\overline{\mathbf{w}}\right)^{\prime} \mathbf{S}^{-1}\left(\mathbf{w}_{i}-\overline{\mathbf{w}}\right)
$$

where $\overline{\mathbf{w}}=\frac{1}{m} \sum_{j=1}^{m} \mathbf{w}_{i}$ and $\mathbf{S}$ is some estimator of the variance-covariance matrix of $\mathbf{w}_{i}$ (Mason and Young [3]). We then plot the $T_{i}^{2}$ statistics, $i=1, \ldots, m$, against $i$ and out-of-control signals will be given for any $T_{i}^{2}$ value exceeding an upper control limit (UCL). For determining the statistical properties of the $T^{2}$-chart it is usually assumed that each of the $\mathbf{w}_{i}$ vectors follows a multivariate normal distribution with common mean vector $\boldsymbol{\mu}$ and covariance matrix $\boldsymbol{\Sigma}$. This assumption is critical to finding the marginal distribution of $T_{i}^{2}$, as discussed in Section 2.3.

In the nonlinear regression model given in Equation $(2), \boldsymbol{\beta}_{i}$ is a $p \times 1$ vector of parameters that determines the curve $f\left(\mathbf{X}_{i}, \boldsymbol{\beta}_{i}\right)$. We employ the the multivariate $T^{2}$ statistic to assess stability of the the $p$ parameters simultaneously, i.e., to evaluate the assumption $\boldsymbol{\beta}_{i}=\boldsymbol{\beta}, i=1, \ldots, m$. We do not employ individual control charts for each of the $p$ nonlinear regression parameters since this may give misleading results due to the built-in correlation structure of the parameter estimators in nonlinear regression.

Once $\hat{\boldsymbol{\beta}}_{i}$ is obtained from each sample in the baseline dataset, we calculate the

average vector $\overline{\hat{\boldsymbol{\beta}}}$ and some corresponding estimate of the covariance matrix, replace $\mathbf{w}_{i}$ with $\hat{\boldsymbol{\beta}}_{i}$ and $\overline{\mathbf{w}}$ with $\overline{\hat{\boldsymbol{\beta}}}$ in Equation (5) to obtain

$$
T_{i}^{2}=\left(\hat{\boldsymbol{\beta}}_{i}-\overline{\hat{\boldsymbol{\beta}}}\right)^{\prime} \mathbf{S}^{-1}\left(\hat{\boldsymbol{\beta}}_{i}-\overline{\hat{\boldsymbol{\beta}}}\right) \text {. }
$$

A large value of $T_{i}^{2}$ indicates an unusual $\hat{\boldsymbol{\beta}}_{i}$, suggesting that the profile for item $i$ 
is out-of-control. In contrast to the traditional use of the $T^{2}$ statistic to monitor a multivariate quality characteristic vector, we employ the $T^{2}$ statistic to monitor the coefficient vectors of the nonlinear regression fit to each individual profile.

There are several choices for the estimator $\mathbf{S}$. Here we discuss the effects of three choices and later discuss under what conditions, if any, each should be used.

The first choice we consider for $\mathbf{S}$ is the sample covariance matrix, given by

$$
\mathbf{S}_{C}=\frac{1}{m-1} \sum_{i=1}^{m}\left(\hat{\boldsymbol{\beta}}_{i}-\overline{\hat{\boldsymbol{\beta}}}\right)\left(\hat{\boldsymbol{\beta}}_{i}-\overline{\hat{\boldsymbol{\beta}}}\right)^{\prime} .
$$

Consequently, the $T_{i}^{2}$ statistics take on the form

$$
T_{C, i}^{2}=\left(\hat{\boldsymbol{\beta}}_{i}-\overline{\hat{\boldsymbol{\beta}}}\right)^{\prime} \mathbf{S}_{C}^{-1}\left(\hat{\boldsymbol{\beta}}_{i}-\overline{\hat{\boldsymbol{\beta}}}\right)
$$

Use of the $T_{C}^{2}$ values was mentioned by Brill [17] in the context of monitoring nonlinear profiles of a chemical product. The advantage of this statistic is that it is very well understood and widely used. However, as was shown by Sullivan and Woodall [18] and Vargas [19], a $T^{2}$ statistic based on $\mathbf{S}_{C}$ is ineffective in detecting sustained shifts in the mean vector during the Phase I period. In fact, it was shown that as the step shift size increased, the power to detect the shift actually decreased.

An alternative choice of $\mathbf{S}$ is one based on successive differences, proposed originally by Hawkins and Merriam [20] and later by Holmes and Mergen [21]. To obtain the estimator, we define $\hat{\mathbf{v}}_{i}=\hat{\boldsymbol{\beta}}_{i+1}-\hat{\boldsymbol{\beta}}_{i}$ for $i=1, \ldots, m-1$ and stack the transpose of these $m-1$ difference vectors into the matrix $\hat{\mathbf{V}}$ as

$$
\hat{\mathbf{V}}=\left[\begin{array}{c}
\hat{\mathbf{v}}_{1}^{\prime} \\
\hat{\mathbf{v}}_{2}^{\prime} \\
\vdots \\
\hat{\mathbf{v}}_{m-1}^{\prime}
\end{array}\right]
$$

The estimator of the variance-covariance matrix is

$$
\mathbf{S}_{D}=\frac{\hat{\mathbf{V}}^{\prime} \hat{\mathbf{V}}}{2(m-1)}
$$


Sullivan and Woodall [18] showed that $\mathbf{S}_{D}$ is an unbiased estimator of the true covariance matrix if the process is stable in Phase I. The resulting $T_{i}^{2}$ statistics are given by

$$
T_{D, i}^{2}=\left(\hat{\boldsymbol{\beta}}_{i}-\overline{\hat{\boldsymbol{\beta}}}\right)^{\prime} \mathbf{S}_{D}^{-1}\left(\hat{\boldsymbol{\beta}}_{i}-\overline{\hat{\boldsymbol{\beta}}}\right)
$$

Sullivan and Woodall [18] and Vargas [19] showed that a $T^{2}$ chart based on values of $T_{D, i}^{2}$ was effective in detecting both a step and ramp shift in the mean vector during Phase I. Sullivan and Woodall [18] also showed that the $T_{D}^{2}$ values are invariant to a full-rank linear transformation on the observations.

Our third choice for $\mathbf{S}$ is a robust estimator of the variance-covariance matrix known as the minimum volume ellipsoid (MVE) estimator, first proposed by Rousseeuw [22] and studied in profile monitoring for Phase I analysis by Jensen, Birch, and Woodall [23]. In our application of the MVE method, we find outlier-robust estimates for both the in-control parameter vector and the variance-covariance matrix based on finding the ellipsoid with the smallest volume that contains at least half of the $\hat{\boldsymbol{\beta}}_{i}$ vectors, $i=1, \ldots, m$. The MVE estimator of $\boldsymbol{\beta}$ is the mean vector of the smallest ellipsoid, and the estimator of the variance-covariance matrix is the sample covariance matrix of the observations within the smallest ellipsoid multiplied by a constant to make the estimator unbiased for multivariate normal data. In a simulation study, Vargas [19] studied the power properties of several different choices of $\mathbf{S}$ in the context of the $T^{2}$ statistic given in Equation (5) and found that the $T^{2}$ statistic based on the MVE estimators of $\boldsymbol{\beta}$ and the variance-covariance matrix was very powerful in detecting multivariate outliers. We denote the MVE estimators of $\boldsymbol{\beta}$ and the covariance matrix by $\hat{\boldsymbol{\beta}}_{M V E}$ and $\mathbf{S}_{M V E}$, respectively. Hence, the fourth choice of $T^{2}$ is

$$
T_{M V E, i}^{2}=\left(\hat{\boldsymbol{\beta}}_{i}-\hat{\boldsymbol{\beta}}_{M V E}\right)^{\prime} \mathbf{S}_{M V E}^{-1}\left(\hat{\boldsymbol{\beta}}_{i}-\hat{\boldsymbol{\beta}}_{M V E}\right), i=1, \ldots, m
$$




\subsection{Control Limits}

The distribution of the $T_{i}^{2}$ statistics for monitoring nonlinear profiles is more complex than in the linear profile case. Recall that the distribution of the parameter estimators in nonlinear regression is difficult to obtain for small sample sizes. Instead we employ the asymptotic distribution (as $n \rightarrow \infty)$ of $\hat{\boldsymbol{\beta}}_{i}, i=1, \ldots, m$. Hence, in order to determine the marginal distribution of $T_{i}^{2}$ in this case, we assume that the sample size, $n$, from each item in the baseline data set is of sufficient size such that the distributions of $\hat{\boldsymbol{\beta}}_{i}, i=1, \ldots, m$ are approximately multivariate normal. The subsequent upper control limits (UCLs) for the multivariate $T^{2}$ control charts are determined based on this normality assumption.

In order to control the overall probability of a false alarm, based on some appropriate UCL, the joint distribution of the $T_{i}^{2}$ values is required. However, these values are correlated since $\overline{\hat{\boldsymbol{\beta}}}$ and $\mathbf{S}$ are used in all $T_{i}^{2}$ statistics $(i=1, \ldots, m)$, thus making the joint distribution of the $T_{i}^{2}$ values difficult to obtain. As an alternative, Mahmoud and Woodall [6] suggested using an approximate joint distribution assuming that the $T_{i}^{2}$ statistics are independent. We let $\alpha$ be the probability of a false alarm for any individual $T_{i}^{2}$ statistic. Then the approximate overall probability of a false alarm for a sample of $m$ items is given by $\alpha_{\text {overall }}=1-(1-\alpha)^{m}$. Thus, for a given overall probability of a false alarm, we use $\alpha=1-\left(1-\alpha_{\text {overall }}\right)^{1 / m}$ in the calculation of UCLs. In their simulation study, Mahmoud and Woodall [6] found that this approximation used to determine the UCLs performed well.

As noted in Tracy, Young, and Mason [24] , Gnanadesikan and Kettenring [25] proved that for a stable process the marginal distribution of $T_{C, i}^{2}$ is proportional to a beta distribution, i.e.,

$$
T_{C, i}^{2} \frac{m}{(m-1)^{2}} \sim B\left(\frac{p}{2}, \frac{m-p-1}{2}\right) .
$$

A formal proof can be found in Chou, Mason, and Young [26] . Note that it is assumed 
that the distribution of $\hat{\boldsymbol{\beta}}_{i}$ is approximately normal. Therefore, an approximate UCL is

$$
U C L_{C}=\frac{(m-1)^{2}}{m} B_{1-\alpha, p / 2,(m-p-1) / 2}
$$

where $B_{1-\alpha, p / 2,(m-p-1) / 2}$ is the $1-\alpha$ quantile of a beta distribution with shape parameters $p / 2$ and $(m-p-1) / 2$.

The marginal distribution of the $T_{D, i}^{2}$ statistic is unknown. However, Williams, Woodall, Birch, and Sullivan [27] gave an approximate distribution based on the chisquared distribution for large sample sizes and an approximate distribution based on the beta distribution for small sample sizes. For large sample sizes, defined by $m>p^{2}+3 p$, the UCL is given by

$$
U C L_{D}=\chi^{2}(1-\alpha, p)
$$

For small sample sizes, defined by $m \leq p^{2}+3 p$ and $p<10$, the UCL is a vector given by

$$
\mathbf{U C L}_{D}=\left(U C L_{1}, U C L_{2}, \ldots, U C L_{m}\right)
$$

where

$$
U C L_{i}=M V(m, i) B E T A_{1-\alpha, \beta(m, p, i), \gamma(m, p, i)}, \quad i=1, \ldots, m
$$

and $\beta(m, p, i)$ and $\gamma(m, p, i)$ are functions of $m, p$, and $i$ that define the two shape parameters for the beta distribution. The specific forms of $\beta(m, p, i)$ and $\gamma(m, p, i)$ given by Williams, et al. [27], are included in the Appendix.

The exact marginal distribution of $T_{M V E, i}^{2}$ is also unknown and intractable. Hence, in order to find the UCL for $T_{M V E, i}^{2}$ we used simulation.

\subsection{Monitoring the Variance}

In addition to checking the stability of each profile in the baseline dataset, it is important to check the stability of the variability about each profile. This is analogous to monitoring the process variance in the standard univariate case. In the 
case of monitoring profiles, we seek to monitor the variability about each profile, or the within-profile variability. Our measure of within-profile variability is the mean squared error (MSE) defined as $M S E_{i}=\sum_{j=1}^{n}\left(y_{i j}-\hat{y}_{i j}\right)^{2} /(n-p)$, where $\hat{y}_{i j}$ is the predicted value of $y_{i j}$ based on the nonlinear regression model in Equation (2). Wludyka and Nelson[28] recommended a method to monitor variances based on an analysisof-means-type test utilizing $S_{i}^{2}=M S E_{i}$. In their paper, $S_{i}^{2}$ is plotted against $i$ with

associated lower- and upper-control limits equal to $\left(L_{\alpha, m, n-p}\right) m \overline{S^{2}}$ and $\left(U_{\alpha, m, n-p}\right) m \overline{S^{2}}$, respectively, where $L$ and $U$ are critical values given in their paper and $\overline{S^{2}}$ is the average of the $S_{i}^{2}$ values, $i=1, \ldots, m$. For large $n$, their approximate upper and lower control limits are $\overline{S^{2}} \pm h_{\alpha, m, \infty} \hat{\sigma}$ where $h$ is a critical value given in Nelson[29] and $\hat{\sigma}=\overline{S^{2}} \sqrt{2(m-1) / m(n-p)}$. The $S_{i}^{2}$ statistics are plotted on a separate control chart to monitor the variance of the error terms and lack of fit simultaneously with a $T^{2}$ control chart for the nonlinear regression parameters. We recommend use of this method when within-profile error terms are independent.

\subsection{Nonparametric Approach}

When a parametric form of a profile would be overly complex, nonparametric procedures may be more appropriate. These include fitting each profile via some smoothing method, such as local polynomial regression, spline smoothing, or wavelets. Walker and Wright[1] give a spline-fitting approach to the vertical density profile (VDP) of particleboard, which we use as an illustration in Section 3. However, these authors discussed using splines to assess variation, not to monitor profiles in a Phase I analysis to check for process stability. Winistorfer, Young, and Walker[30] illustrated the use of splines to model the VDP of oriented strandboard generated from a $3^{2}$ factorial design with 3 replicates. However, their spline-fitting method is used in the context of comparing profiles among differing experimental conditions, not monitoring 
profiles in a Phase I or Phase II analysis.

For the case of a single explanatory variable, we denote the nonparametric fit of profile $i$ by $\dot{y}_{i j}$, for the corresponding value of the explanatory variable equal to $x_{j}$, $j=1, \ldots, n$. The general nonparametric approach to monitoring profiles in Phase I analysis is to establish a "baseline" curve with which to compare all other curves. A natural choice of baseline profile is the average estimated profile across all $m$ profiles, denoted by $\widetilde{y}_{j}=\sum_{i=1}^{m} \dot{y}_{i j} / m, j=1, \ldots, n$. Once a baseline curve is found, some appropriate distance metric can be used to measure how "different" each individual curve is from the baseline. Researchers at Boeing [31] proposed the following three metrics:

1. $M_{i 1}=\operatorname{sign}\left(\max _{j}\left|\dot{y}_{i j}-\widetilde{y}_{i j}\right|\right)$

2. $M_{i 2}=\sum_{j=1}^{n}\left|\dot{y}_{i j}-\widetilde{y}_{i j}\right|$

3. $M_{i 3}=\sum_{j=1}^{n}\left|\dot{y}_{i j}-\widetilde{y}_{i j}\right| / m$.

The three metrics, $M_{i 1}, M_{i 2}$, and $M_{i 3}$, are referred to as the maximum deviation, sum of absolute deviations, and the mean absolute deviation, respectively. Further, it may be of interest to compute the absolute value of $M_{i 1}$, which obviously reflects the magnitude of the dissimilarity between $\dot{y}_{i j}$ and $\widetilde{y}_{i j}$ disregarding the direction of dissimilarity. We denote this metric by $M_{i 4}$. Other metrics are proposed in Gardner, et. al.[32], who note that metrics can be defined to detect changes in profiles resulting from particular known process faults. One of these metrics is the sum of squared differences between each estimated profile and the average profile, denoted $M_{i 5}=\sum_{j=1}^{n}\left(\dot{y}_{i j}-\widetilde{y}_{i j}\right)^{2}$. For a given metric, one plots the metric value for profile $i$ against $i(i=1, \ldots, m)$ and checks for unusual observations. Researchers at Boeing [31] suggested using a standard univariate $I$-chart on the metrics to establish control limits. The method of smoothing splines with several dissimilarity metrics is 
illustrated in Section 3.

\section{EXAMPLE}

In this example to illustrate the application of the various approaches we use the vertical density profile data from Walker and Wright [1], available at the website http://filebox.vt.edu/users/bwoodall/VDP\%20nonlinear\%20profile\%20data.txt. In the manufacture of particleboard, the density properties of the finished boards are quality characteristics that are monitored through time. It is well known that the density (in pounds per $\mathrm{ft}^{3}$ ) near the core of a particleboard is much less than the density at the top and bottom faces of a board (see Young, Winistorfer, and Wang [33]). The standard sampling procedure calls for a laser-aided density measuring device to scan fixed vertical depths of a board and record the density at each depth. Since the depths are fixed for each board, we denote the depth $x_{i j}$ by simply $x_{j}$. Density measurements for this dataset were taken at depths of $x_{j}=(0.002) j$ inches, $j=0,1,2, \ldots, 313$. Correspondingly, a sequence of ordered pairs, $\left(x_{j}, y_{i j}\right), j=1, \ldots, n$, results for board $i$ and forms a vertical density profile (VDP) of the board. A baseline sample of twenty-four particleboards was measured in this way, and the twenty-four profiles are illustrated in Figure 1.

\section{[Insert Figure 1 about here.]}

Young, Winistorfer, and Wang [33] introduced a statistical method to monitor VDP data. With their method, one summarizes the density measurements into three average density measurements: one near the core and one near each face. The three averages are the quality characteristics that are subsequently monitored using a standard multivariate $T^{2}$ control chart. With this method one basically summarizes each nonlinear profile into only three numbers with a corresponding loss of information. 
An alternative approach without such a considerable loss of information is to model the profiles themselves parametrically. The nonlinear function we use to model profile $i$ is a "bathtub" function given by

$$
f\left(x_{i j}, \boldsymbol{\beta}\right)=\left\{\begin{array}{cc}
a_{1}\left(x_{i j}-c\right)^{b_{1}}+d \quad x_{j}>c \\
a_{2}\left(-x_{i j}+c\right)^{b_{2}}+d \quad x_{j} \leq c
\end{array} \quad i=1, \ldots, m ; j=1, \ldots, n,\right.
$$

where $\boldsymbol{\beta}=\left(a_{1}, a_{2}, b_{1}, b_{2}, c, d\right)$. One advantage of this nonlinear model is the interpretability of the model parameters. For example, $a_{1}, a_{2}, b_{1}$, and $b_{2}$ determine the "flatness", $c$ is the center, and $d$ is the bottom, or the "level" of the curve. Differing values of $a_{1}$ and $a_{2}$ or different values of $b_{1}$ and $b_{2}$ allow for an asymmetric curve about the center $c$. Figure 2 contains the "bathtub" function fit to board 1 from the VDP data.

\section{[Insert Figure 2 about here.]}

The profile of board 1 is well-modeled by this parametric fit $\left(R^{2}>0.9999\right)$. For each of the twenty-four boards in the baseline sample we fit the nonlinear model in Equation (11), and calculated the $T_{C}^{2}, T_{D}^{2}$, and $T_{M V E}^{2}$ statistics of Equations (6), (7), and (8), respectively. based on the $\hat{\boldsymbol{\beta}}_{i}$ values. Parameter estimates for each of the twenty-four boards and the corresponding $T^{2}$ statistics are given in Table 1 . We plot the six parameter estimates for each of the twenty-four boards in Figure 3.

\section{[Insert Table 1 about here.] \\ [Insert Figure 3 about here.]}

The control limits for the $T_{C}^{2}, T_{D}^{2}$ statistics are calculated from Equations 9 and 10, respectively. We simulated the UCL for the $T_{M V E}^{2}$ statistic to achieve an overall probability of a signal equal to 0.05 for $m=24$ boards. In our simulation, we sampled from a multivariate normal distribution of dimension six, mean vector zero, and 
variance-covariance matrix $I$, since the in-control performance of the methods does not depend on the assumed in-control parameter vector or the variance-covariance matrix. We repeated our simulation 200,000 times, giving a standard error for the estimated control limits less than 0.0005 . The UCL values are 14.72 and 65.37 , for the $T_{C}^{2}$ and $T_{M V E}^{2}$ control charts, respectively, and the control limit vector for the $T_{D}^{2}$ control chart is given in Table 1.

In Phase I analysis, we are interested in identifying "outlying" or out-of-control boards or a shift in the process which might affect the estimation of in-control parameters. We compared the four $T^{2}$ control charts for assessing process stability and identifying outlying profiles. In Figure 4 we illustrate all four $T^{2}$ control charts for the VDP data.

\section{[Insert Figure 4 about here.]}

Both the $T_{C}^{2}$ and the $T_{D}^{2}$ control charts indicate that board 15 has the only outof-control profile, although the profile for board 18 is borderline. Note that the $T_{D}^{2}$ statistic accentuates the same outlying observations of the $T_{C}^{2}$ chart, but has a larger UCL. As discussed in Sullivan and Woodall [18], the $T_{C}^{2}$ control chart has greater power to detect isolated outlying observations than the $T_{D}^{2}$ control chart based on the successive differences variance-covariance matrix estimator, however the $T_{D}^{2}$ chart is better for detecting a sustained shift in the mean vector. For this dataset, there is no apparent sustained shift in the regression parameter vector.

The $T_{M V E}^{2}$ control chart based on the minimum variance ellipsoid estimator indicates that boards $4,9,15,18$, and 24 have outlying profiles. The most pronounced outlier is board 15 , which both the $T_{C}^{2}$ and $T_{D}^{2}$ charts also indicated as the most severe outlier. As shown by Vargas[19], the $T_{M V E}^{2}$ control chart is very powerful in detecting multivariate outliers. Investigating the table of parameter estimates for these boards, given in Table 1, it seems reasonable that the boards 15 and 18 are outliers, with 
boards 4,9 , and 24 worthy of further investigation.

As discussed in Section 2.5, an alternative approach to modeling the profiles with a parametric curve is to employ nonparametric smoothing techniques to model the profiles. Walker and Wright[1] employed spline smoothing with 16 degrees of freedom to model the twenty-four boards of the VDP data. We replicated their spline fits to each profile. After obtaining the spline fits to each profile, $\dot{y}_{i j}, i=1, \ldots, m ; j=$ $1, \ldots, n$, the average spline, $\widetilde{y}_{j}$, is calculated. For example, the spline fit to board 1 and the average spline are illustrated in Figure 5.

[Insert Figure 5 about here.]

The spline fit with 16 degrees of freedom provides a concise summary of the shape of the profile from board 1 . The average spline fit is systematically lower than the spline fit to board 1. In order to determine which boards are in-control we calculated dissimilarity metrics as given in Section 2.5. Since the metrics $M_{i 2}$ and $M_{i 3}$ differ only by a constant, it is not helpful to consider both metrics simultaneously. Instead we calculate the metrics $M_{i 1}, M_{i 3}, M_{i 4}$, and $M_{i 5}$, and then employ an $I$-chart based on the moving range to establish control limits, as suggested by researchers at Boeing[31]. We plot each metric versus $i$ with associated control limits to obtain control charts. The four charts are given in Figure 6.

\section{[Insert Figure 6 about here.]}

The charts based on metrics $M_{i 1}$ and $M_{i 4}$ both give the same conclusion, that all the profiles of the boards are in-control. This is not surprising since $M_{i 4}$ is the absolute value of $M_{i 1}$, but both are given for illustrative purposes. The most extreme value of the metrics came from board 14 , with values of $M_{14,1}=-5.79$ and $M_{14,4}=5.79$. This value represents the maximum (absolute) deviation of the spline fit to board 14 from the average spline fit. 
Similarly, the charts based on metrics $M_{i 3}$ and $M_{i 5}$ both give the same conclusion, that the profile for board 6 is out-of-control. Referring to Figure 1, board 6 is the one with the profile that is consistently lower than all other boards. The next most extreme value of the two metrics is that of board 3, although it does not give an out-of-control signal. Again, referring to Figure 1, board 3 is the one with the profile that is consistently higher than all the other boards. It is apparent that these two metrics measure how consistently different each profile is from the average profile across the depth values, whereas metrics $M_{i 1}$ and $M_{i 4}$ measure the greatest extent to which a profile is from the average at any particular depth value. It is important to note that the results for the control charts on the metrics (Figure 6) do not show the same results as the control charts based on the regression estimators in Figure 4. If the profile can be adequately represented by a parametric model, then this, in general, will lead to more effective charts.

In addition to monitoring the regression parameter vectors of the profiles in a Phase I analysis, we should monitor the variation about the profiles to check for stability. As mentioned in Section 2.4, we recommend using the methods of Wludyka and Nelson [28] to monitor the variance $\sigma^{2}$. Use of their method is appropriate when the error terms within a profile are independent. In our VDP example, however, the within-profile density measurements are spatially correlated. A more appropriate control chart in this case to monitor the process variance $\sigma^{2}$ is a topic for further research.

\section{AUTOCORRELATION}

Engineering applications that give rise to nonlinear profile data may lead to autocorrelated error terms. A common source of autocorrelated errors is the spatial or serial manner in which data are collected. The VDP data, for example, is spatially 
correlated because the density measurements are taken at close intervals along the vertical depth of the particleboard. On the other hand, some nonlinear profiles may have independent error terms. One example of this is typical dose-response data where several doses of a particular drug are administered to different subjects and their responses are measured. The subsequent error terms in the nonlinear dose-response curve are typically assumed to be independent.

When the error terms are autocorrelated due to either serial, spatial or any other effects, the correlation structure should be taken into account in the analysis. Failure to do so might yield misleading results in some cases, particularly with the control chart to monitor $\sigma^{2}$. In our example, given in Section 3, we estimated parameters of a nonlinear regression model for each board. For our nonlinear model we assumed that the errors $\epsilon_{i j}$ are i.i.d. For the VDP data, it may be reasonable to assume that the $\epsilon_{i j}$ are correlated. If this is the case, perhaps an alternative approach would be to employ either nonlinear mixed model methods or generalized estimating equations (GEE) methodology. Both methods can be used to estimate the mean function, or profile, while accounting for autocorrelation in the error structure. In the presence of an autocorrelated error term, Jensen and Birch [34] show that profile analysis based on a nonlinear mixed model offers an improved control chart performance over a nonlinear profile analysis assuming an independent error term. A more detailed treatment of these methods can be found in Schabenberger and Pierce [14] and Hardin and Hilbe [35]. In the context of analyzing nonlinear profiles for Phase I applications, this approach is a topic that requires further investigation.

\section{DISCUSSION}

In Phase I, we are interested in identifying outlying observations as well as identifying step or ramp shifts in the mean vector over time. As shown by Vargas [19], the 
robust variance-covariance matrix and mean vector estimators employed in the $T_{M V E}^{2}$ statistic are very powerful in detecting multivariate outliers, but are not powerful in detecting a step shift. However, the opposite is true of the $T_{D}^{2}$ statistic. As shown by Sullivan and Woodall[18], the $T_{D}^{2}$ chart is powerful in detecting a step shift, but not powerful in detecting multivariate outliers. One possible alternative is to employ both the $T_{D}^{2}$ and $T_{M V E}^{2}$ charts simultaneously, the former chart sensitive to step shifts and the latter sensitive to outliers. However, in examining both charts simultaneously, one must be cautious of inflating the false alarm probability. This approach is also a topic for further research.

We have not given a detailed treatment of the nonparametric approaches to monitoring profiles discussed in Section 2.5. Rather, we have only described some methods that have been proposed and then illustrated their use with the VDP data. Some issues that need to be addressed, for example, are the best nonparametric estimation technique for a given scenario, the best metrics to apply, the strengths and weaknesses of each metric, and the distributional properties of the metrics in order to establish valid control limits.

The field of profile monitoring using control charts has potential to extend statistical process control to a wide variety of engineering and pharmaceutical applications. With the increasing ease and efficiency in which processes and products can be measured, there is a need for statistical methodology to be developed which can accommodate the growing needs of industry. We have encountered a number of engineering applications in which a response curve is needed to assess quality. In some cases, the shape of the response curve can be well-represented by a parametric nonlinear regression function. In this paper we have developed control chart methodology to monitor such nonlinear profiles for Phase I applications. When a profile cannot be easily described by a parametric function, nonparametric methods may be applied. 


\section{APPENDIX}

The function for $\beta(m, p, i)$ is

$$
\beta(m, p, i)=I_{\{i=1, m\}}\left(\frac{p}{2}-\frac{1}{a_{11}\left(m-b_{11}\right)}\right)+I_{\{i=2, \ldots, m-1\}}\left(a_{12} p+b_{12}\right),
$$

and the function for $\gamma(m, p, i)$ is given by

$$
\gamma(m, p, i)=I_{\{i=1, m\}} a_{21}+I_{\{i=2, \ldots, m-1\}}\left[a_{22}\left(i-\frac{m+1}{2}\right)^{2}+b_{22}\right],
$$

where

$$
\begin{aligned}
I_{\{i=1, m\}} & =\left\{\begin{array}{cc}
1 & \text { if } i=1 \text { or } i=m \\
0 & \text { otherwise }
\end{array}\right. \\
I_{\{i=2, \ldots, m-1\}} & =\left\{\begin{array}{cc}
1 & \text { if } 2 \leq i \leq m-1 \\
0 & \text { otherwise }
\end{array}\right. \\
a_{11} & =6.356 e^{-0.825 p}+0.06 \\
b_{11} & =0.5564 p+0.9723 \\
a_{12} & =0.54-0.25 e^{-0.25(m-15)} \\
b_{12} & =-0.085+0.2 e^{-0.2(m-22)} \\
a_{21} & =(-0.5 m+2) p+\frac{1}{3}(m+3)(m-5) \\
a_{22} & =0.99+0.38 e^{0.38(p-13.5)}-\frac{1}{0.25 e^{-0.25(p-10)}\left(m-11+\frac{(p-7)^{2}}{3}\right)} \\
b_{22} & =\left(0.07 e^{-0.07(m-42)}-1.95\right) p+0.0833 m^{2}
\end{aligned}
$$




\section{REFERENCES}

1. Walker, E., and Wright, S. (2002), "Comparing Curves Using Additive Models," Journal of Quality Technology, 34, pp. 118-129.

2. Fuchs, C., and Kenett, R. S. (1998), Multivariate Quality Control: Theory and Applications, New York: Marcel Dekker.

3. Mason, R. L., and Young, J. C. (2002), Multivariate Statistical Process Control with Industrial Applications, Philadelphia: SIAM.

4. Woodall, W. H.; Spitzner, D. J.; Montgomery, D. C.; and Gupta, S. (2004). "Using Control Charts to Monitor Process and Product Quality Profiles". Journal of Quality Technology 36, pp. 309-320.

5. Williams, J. D., Birch, J. B., Woodall, W. H., and Ferry, N. M. (2006), "Statistical Monitoring of Heteroscedastic Dose-Response Profiles from High-Throughput Screening," Journal of Agricultural, Biological, and Environmental Statistics, To Appear.

6. Mahmoud, M. A., and Woodall, W. H. (2004), "Phase I Analysis of Linear Profiles with Calibration Applications," Technometrics, 46, pp. 377-391.

7. Gupta, S., Montgomery, D. C., and Woodall, W. H. (2006), "Performance Evaluation of Two Methods for Online Monitoring of Linear Calibration Profiles", International Journal of Production Research, 44, pp. 1927-1942.

8. Kang, L., and Albin, S. L. (2000), "On-line Monitoring When the Process Yields a Linear Profile," Journal of Quality Technology, 32, pp. 418-426.

9. Kim, K., Mahmoud, M. A., and Woodall, W. H. (2003), "On the Monitoring of Linear Profiles," Journal of Quality Technology, 35, pp. 317-328. 
10. Jensen, D. R., Hui, Y. V., and Ghare, P. M. (1984), "Monitoring an Inputoutput Model for Production. I. The Control Charts," Management Science, 30, pp. 1197-1206.

11. Ding, Y., Zeng, L., and Zhou, S. (2006), "Phase I Analysis for Monitoring Nonlinear Profiles in Manufacturing Processes," Journal of Quality Technology, 38, pp. 199-216.

12. Sullivan, J. H. (2002), "Detection of Multiple Change Points from Clustering Individual Observations," Journal of Quality Technology, 34, pp. 371-383.

13. Myers, R. H. (1990), Classical and Modern Regression With Applications, second edition, Belmont, California: Duxbury Press.

14. Schabenberger, O. and Pierce F. J. (2002), Contemporary Statistics for the Plant and Soil Sciences, Boca Raton, Florida: CRC Press.

15. Gallant, A. R. (1987), Nonlinear Statistical Models, New York: Wiley.

16. Seber, G. A. F., and Wild, C. J. (1989), Nonlinear Regression, New York: Wiley.

17. Brill, R. V. (2001), "A Case Study for Control Charting a Product Quality Measure that is a Continuous Function Over Time," Presentation at the $47^{\text {th }}$ Annual Fall Technical Conference, Toronto, Ontario.

18. Sullivan, J. H., and Woodall, W. H. (1996), "A Comparison of Multivariate Control Charts for Individual Observations," Journal of Quality Technology, 28, pp. 398-408.

19. Vargas J. A. (2003), "Robust Estimation in Multivariate Control Charts for Individual Observations," Journal of Quality Technology, 35, pp. 367-376. 
20. Hawkins, D. M., and Merriam, D. F. (1974), "Zonation of Multivariate Sequences of Digitized Geologic Data," Mathematical Geology, 6, pp. 263-269.

21. Holmes, D. S, and Mergen, A. E. (1993), "Improving the Performance of the $T^{2}$ Control Chart," Quality Engineering, 5, pp. 619-625.

22. Rousseeuw, P. J. (1984), "Least Median of Squares Regression," Journal of the American Statistical Association, 79, pp. 871-880.

23. Jensen, W. A., Birch, J.B., and Woodall, W.A. (2007), "High Breakdown Estimation Methods for Phase I Multivariate Control Charts", Quality and Reliability Engineering International. In press.

24. Tracy, N. D., Young, J. C., and Mason, R. L. (1992), "Multivariate Control Charts for Individual Observations," Journal of Quality Technology, 24, pp. 88-95.

25. Gnanadesikan, R., and Kettenring, J. R. (1972), "Robust Estimates, Residuals, and Outlier Detection with Multiresponse Data," Biometrics, 28, pp. 81-124.

26. Chou, Y. -M., Mason, R. L., and Young J. C. (1999), "Power Comparisons for a Hotelling's $T^{2}$ Statistic," Communications in Statistics, Part B - Simulation and Computation, 28, pp. 1031-1050.

27. Williams, J. D., Woodall, W. H., Birch, J. B., and Sullivan, J. H. (2006), "On the Distribution of $T^{2}$ Statistics Based on Successive Differences," Journal of Quality Technology 38, pp. 217-229.

28. Wludyka, P. S., and Nelson, P. R. (1997), "An Analysis-of-Means-Type Test for Variances From Normal Populations," Technometrics, 39, pp. 274-285. 
29. Nelson, L. S. (1983), "Exact Critical Values for the Analysis of Means," Journal of Quality Technology, 15, pp. 40-44.

30. Winistorfer, P. M., Young, T. M., and Walker, E. (1996), "Modeling and Comparing Vertical Density Profiles," Wood and Fiber Science, 28, pp. 133-141.

31. Boeing Commercial Airplane Group, Materiel Division, Procurement Quality Assurance Department (1998), Advanced Quality System Tools, AQS D1-90001, The Boeing Company: Seattle, WA.

32. Gardner M. M., Lu, J. -C., Gyuresik, R. S., Wortman, J. J., Hornung, B. E., Heinisch, H. H., Rying, E. A., Rao, S., Davis, J. C., Mozumder, P. K. (1997), "Equipment Fault Detection Using Spatial Signatures," IEEE Transactions on Components, Packaging, and Manufacturing Technology - Part C, 20, pp. 295304.

33. Young, T. M., Winistorfer, P. M., and Wang, S. (1999), "Multivariate Control Charts of MDF and OSB Vertical Density Profile Attributes," Forest Products Journal, 49(5), pp. 79-86.

34. Jensen, W.A. and Birch, J. B. (2006), "Profile Monitoring via Nonlinear Mixed Models". Virginia Tech Department of Statistics Technical Report 06-04. http://www.stat.org.vt.edu/dept/web-e/tech_reports/TechReport06-4.pdf. Submitted for publication.

35. Hardin, J. W., and Hilbe, J. M. (2003), Generalized Estimating Equations, Boca Raton, Florida: CRC Press. 


\section{Author Biographies}

James D. Williams is Statistical Leader at General Electric Global Research in Schenectady, NY. He holds a BA in Mathematics Education (1999) and a MS in Statistics (2001) from Brigham Young University, and a PhD in Statistics (2004) from Virginia Tech. His research interests include profile monitoring, multivariate statistical process control, regression modeling, and financial risk modeling. He is a recipient of the Richard A. Freund International Scholarship (2003) from the American Society for Quality, the Mary G. Natrella Scholarship (2003) from the Quality \& Productivity Section of the American Statistical Association, and the Ellis R. Ott Scholarship (2004) from the Statistics Division of the American Society for Quality. He is currently serving as an elected officer of the Quality \& Productivity Section of the American Statistical Association.

William H. Woodall is Professor of Statistics at Virginia Tech. He holds a BS in Mathematics from Millsaps College (1972), and a MS (1974) and PhD (1980) in Statistics from Virginia Tech. His research interests are statistical quality control and improvement, all aspects of control charting, public health surveillance, and critiques of fuzzy logic. Dr. Woodall is a former editor of the Journal of Quality Technology (2001-2003), associate editor of Technometrics (1987-1995), and serves on the editorial review board for the Journal of Quality Technology (1988-present). He is the recipient of the Shewhart Medal (2002), Jack Youden Prize (1995, 2003) and the Brumbaugh Award (2000). He is a Fellow of the American Statistical Association, American Society for Quality, and an elected member of the International Statistical Institute.

Jeffrey B. Birch is Professor of Statistics and Director of Graduate Programs at Virginia Tech, where he has been a faculty member since 1977. Dr. Birch received his MS (1970) and PhD (1977) degrees in biostatistics from the University of Washington. His area of primary research includes most aspects of regression analysis including 
robust regression, nonparametric and semiparametric regression, and profile monitoring. Dr. Birch is a former associate editor of Biometrics (1989-1993). 
Table 1: Estimated Parameter Values and $T^{2}$ Statistics for the VDP Data

\begin{tabular}{|c|c|c|c|c|c|c|c|c|c|c|}
\hline Board & $\hat{a}_{1}$ & $\hat{a}_{2}$ & $\hat{b}_{1}$ & $\hat{b}_{2}$ & $\hat{\boldsymbol{c}}$ & $\hat{d}$ & $T_{C}^{2}$ & $T_{D}^{2}$ & $T_{M V E}^{2}$ & $U C L_{D}$ \\
\hline 1 & 6560 & 3259 & 5.63 & 4.40 & 45.98 & 0.29 & 2.65 & 1.91 & 6.00 & 27.88 \\
\hline 2 & 470 & 291 & 3.01 & 2.74 & 42.08 & 0.32 & 7.56 & 5.27 & 6.97 & 22.29 \\
\hline 3 & 1812 & 2871 & 3.99 & 5.02 & 47.66 & 0.34 & 5.83 & 7.17 & 8.64 & 22.27 \\
\hline 4 & 6171 & 15009 & 4.25 & 7.39 & 46.63 & 0.39 & 12.21 & 17.28 & 1131.81 & 22.24 \\
\hline 5 & 4963 & 2251 & 5.14 & 4.20 & 43.43 & 0.30 & 1.65 & 2.27 & 2.88 & 22.21 \\
\hline 6 & 4556 & 3758 & 5.28 & 4.72 & 40.13 & 0.30 & 8.49 & 13.03 & 9.83 & 22.17 \\
\hline 7 & 5542 & 3815 & 5.25 & 5.00 & 44.15 & 0.31 & 2.15 & 3.49 & 3.58 & 22.12 \\
\hline 8 & 3664 & 2979 & 4.89 & 4.41 & 44.06 & 0.30 & 0.79 & 0.97 & 2.69 & 22.07 \\
\hline 9 & 28041 & 8872 & 7.58 & 4.95 & 43.22 & 0.26 & 4.62 & 7.10 & 385.03 & 22.01 \\
\hline 10 & 1640 & 1207 & 4.17 & 3.39 & 41.84 & 0.28 & 4.30 & 5.05 & 4.61 & 21.95 \\
\hline 11 & 3492 & 1031 & 5.82 & 3.17 & 46.06 & 0.25 & 8.66 & 8.95 & 10.00 & 21.91 \\
\hline 12 & 915 & 750 & 3.45 & 3.52 & 44.37 & 0.32 & 1.80 & 1.99 & 2.22 & 21.88 \\
\hline 13 & 989 & 1392 & 3.58 & 4.05 & 45.47 & 0.32 & 3.42 & 4.42 & 5.18 & 21.88 \\
\hline 14 & 1474 & 620 & 4.82 & 3.29 & 42.52 & 0.27 & 3.28 & 4.50 & 7.04 & 21.91 \\
\hline 15 & 129068 & 5420 & 12.40 & 3.33 & 45.90 & 0.15 & 21.45 & 22.18 & 17018.91 & 21.95 \\
\hline 16 & 10166 & 3822 & 5.83 & 4.86 & 44.19 & 0.30 & 3.83 & 5.60 & 12.93 & 22.01 \\
\hline 17 & 1483 & 603 & 4.07 & 3.26 & 44.83 & 0.30 & 2.30 & 2.53 & 2.36 & 22.07 \\
\hline 18 & 31156 & 31069 & 7.70 & 5.94 & 46.46 & 0.27 & 14.55 & 19.75 & 8221.00 & 22.12 \\
\hline 19 & 418 & 198 & 3.22 & 2.67 & 42.84 & 0.30 & 4.58 & 3.90 & 5.16 & 22.17 \\
\hline 20 & 3207 & 4741 & 4.88 & 5.02 & 44.45 & 0.30 & 5.34 & 5.59 & 34.00 & 22.21 \\
\hline 21 & 672 & 773 & 3.37 & 3.37 & 44.46 & 0.31 & 2.64 & 3.42 & 2.79 & 22.24 \\
\hline 22 & 3520 & 1807 & 5.10 & 4.01 & 45.52 & 0.29 & 1.71 & 1.37 & 1.73 & 22.27 \\
\hline 23 & 1979 & 845 & 4.24 & 3.66 & 45.53 & 0.32 & 4.45 & 4.85 & 7.38 & 22.29 \\
\hline 24 & 6095 & 26778 & 5.41 & 6.67 & 44.46 & 0.31 & 9.75 & 10.55 & 6676.21 & 27.88 \\
\hline
\end{tabular}


Figure 1: Vertical Density Profile (VDP) of 24 Particleboards

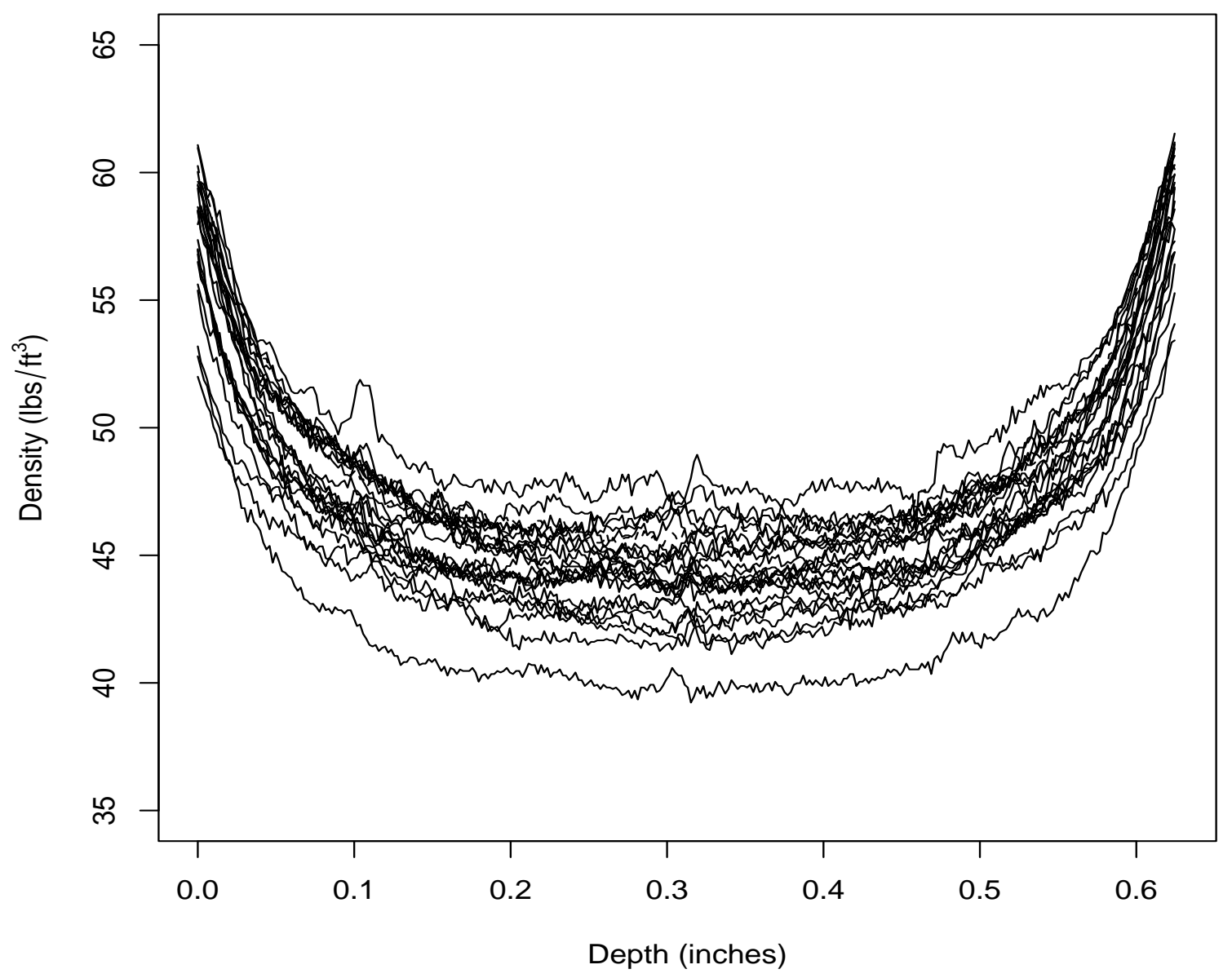


Figure 2: "Bathtub" Function Fit to Board 1

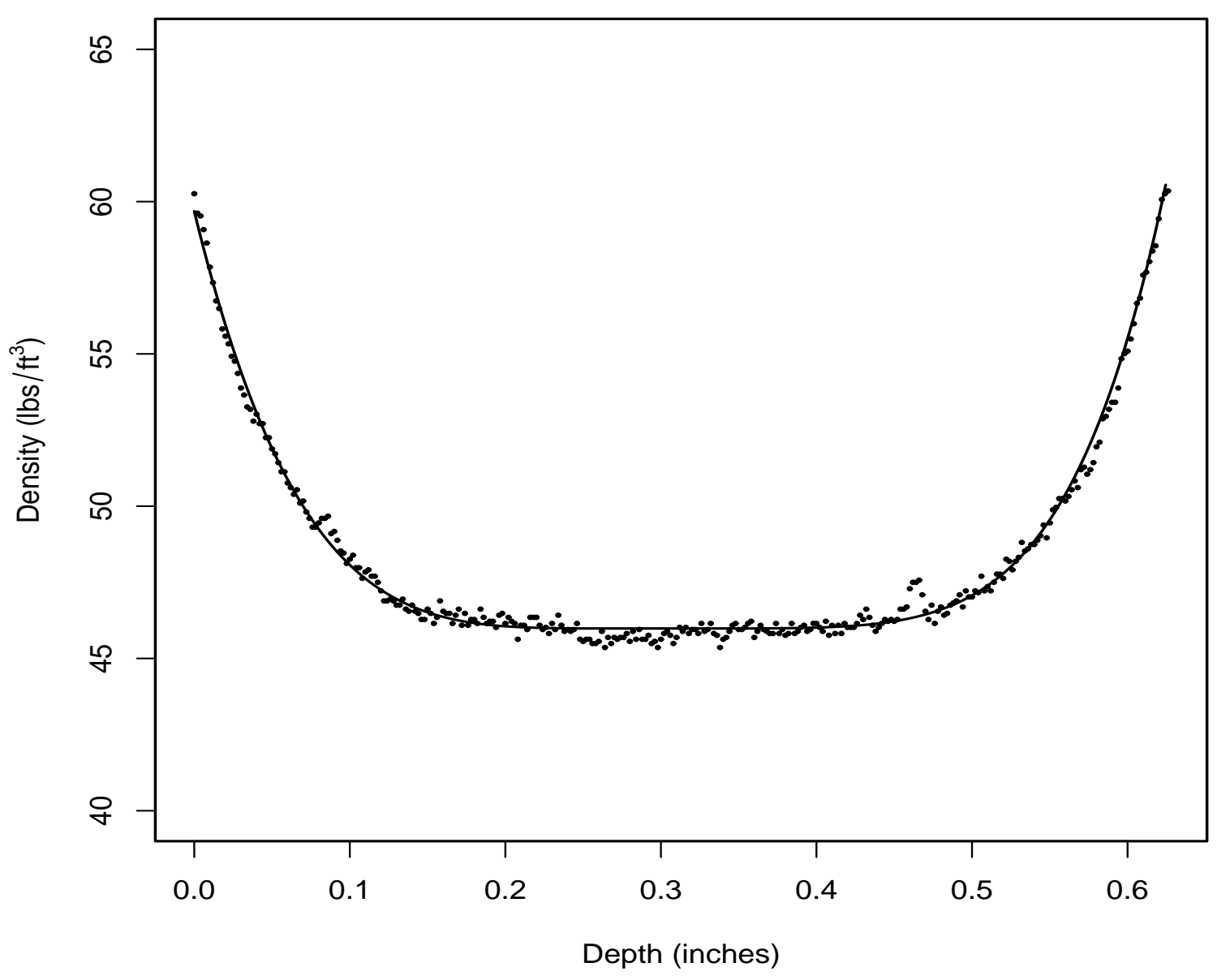


Figure 3: Nonlinear Regression Parameter Estimates $a_{1}, a_{2}, b_{1}, b_{2}, c$, and $d$ by Board for the VDP Data
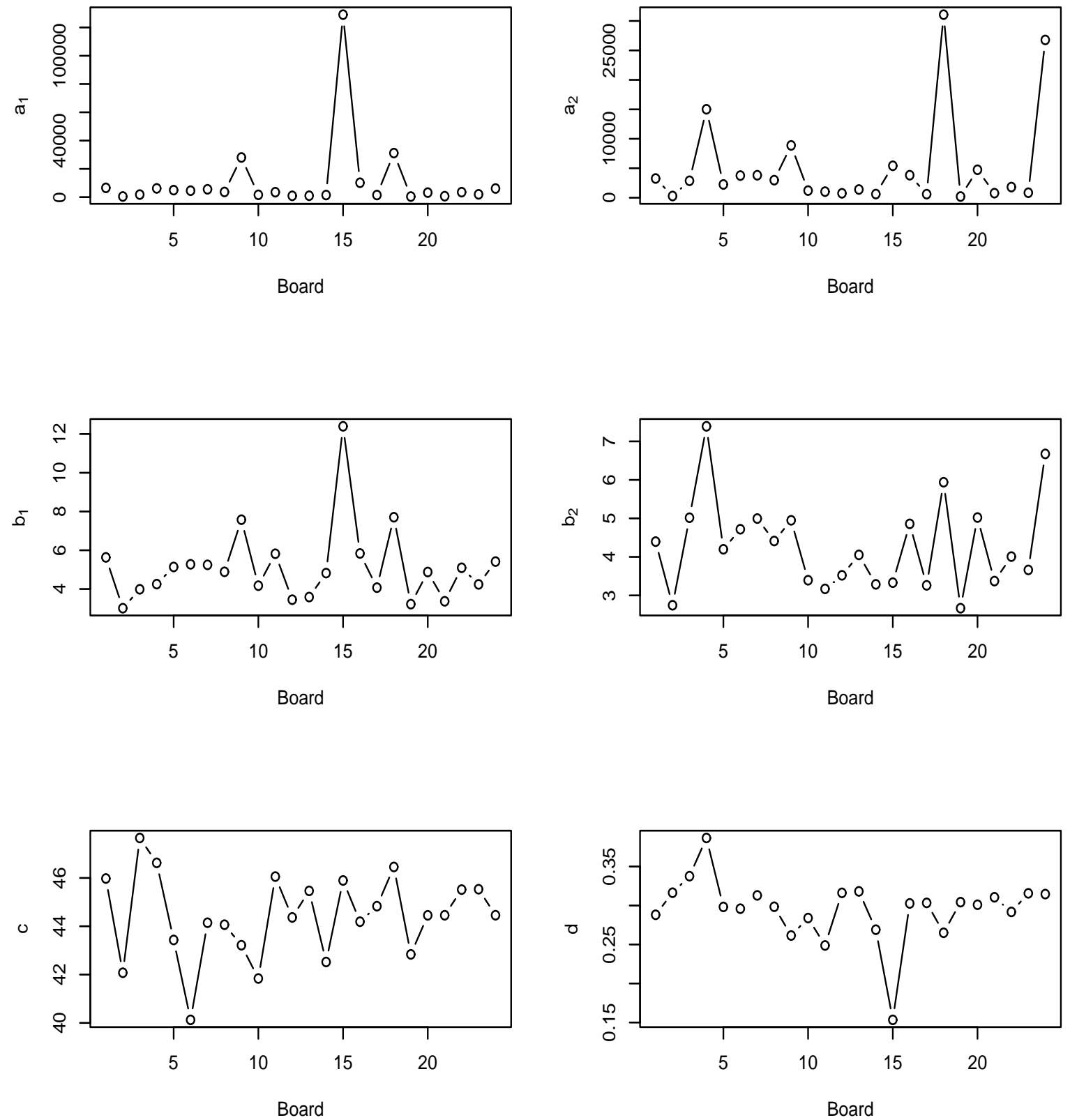
Figure 4: The $T^{2}$ Control Charts for the VDP Data. (a) The $T_{C}^{2}$ control chart based on the sample covariance matrix, (b) $T_{D}^{2}$ control chart based on the successive differences estimator, and (c) $T_{M V E}^{2}$ control chart based on the minimum volume ellipsoid.

(a)

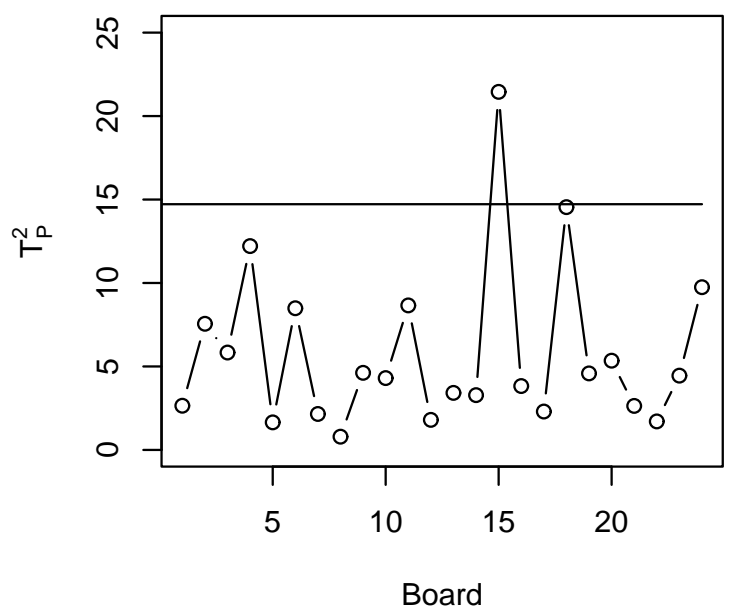

(c)

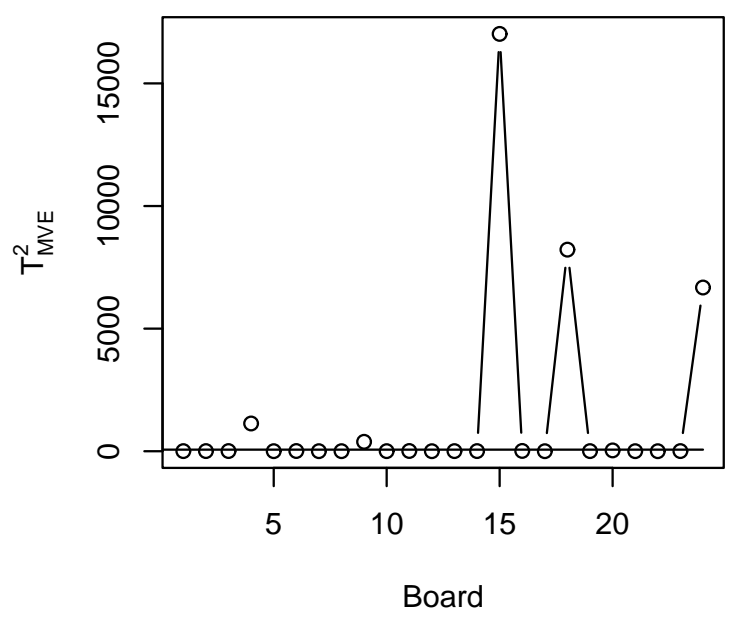

(b)

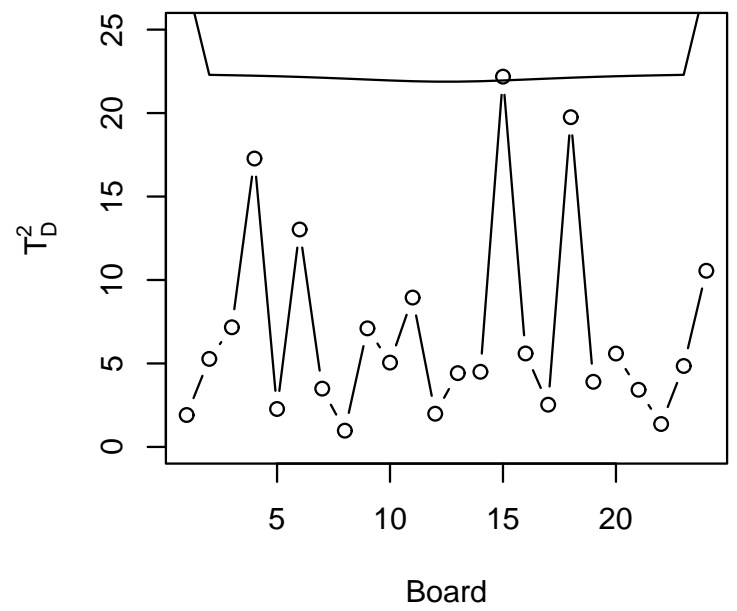


Figure 5: Spline Fit of Board 1 (Above) and Average Spline (Below) for the VDP Data

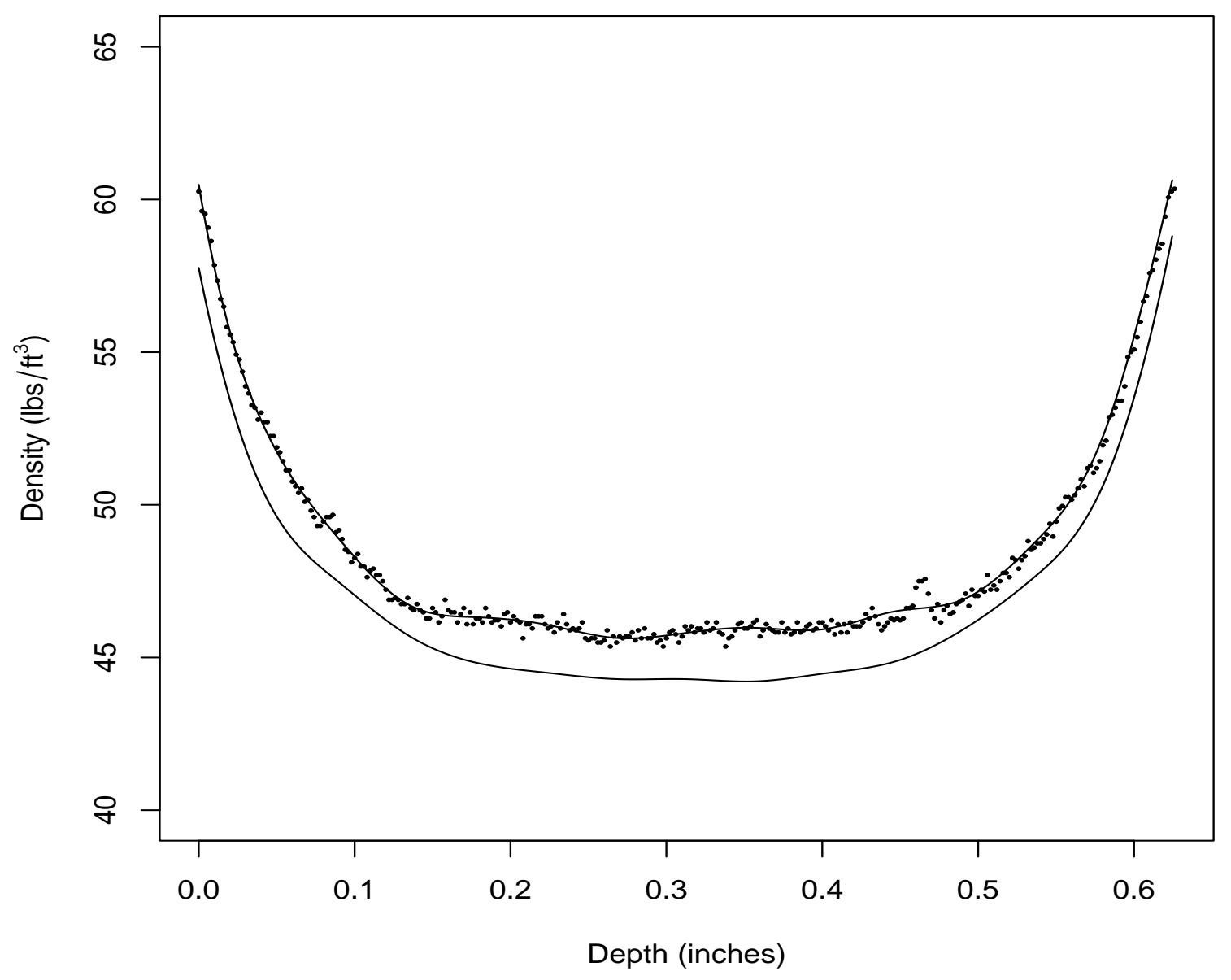


Figure 6: Control Charts on Metrics: (a) $M_{i 1}$, the Maximum Deviation, (b) $M_{i 3}$, the Maximum Absolute Deviation, (c) $M_{i 4}$, the Sum of Squared Differences, and (d) $M_{i 5}$, the Mean Absolute Deviation for the VDP Data

(a)

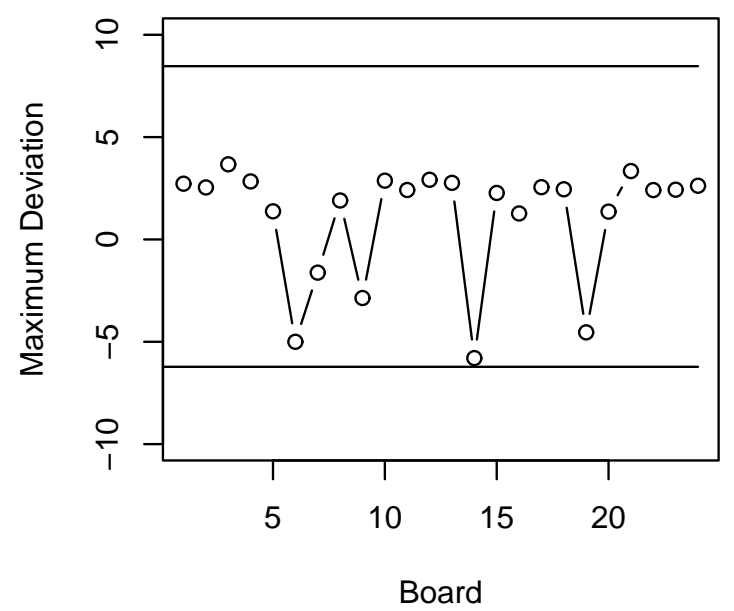

(c)

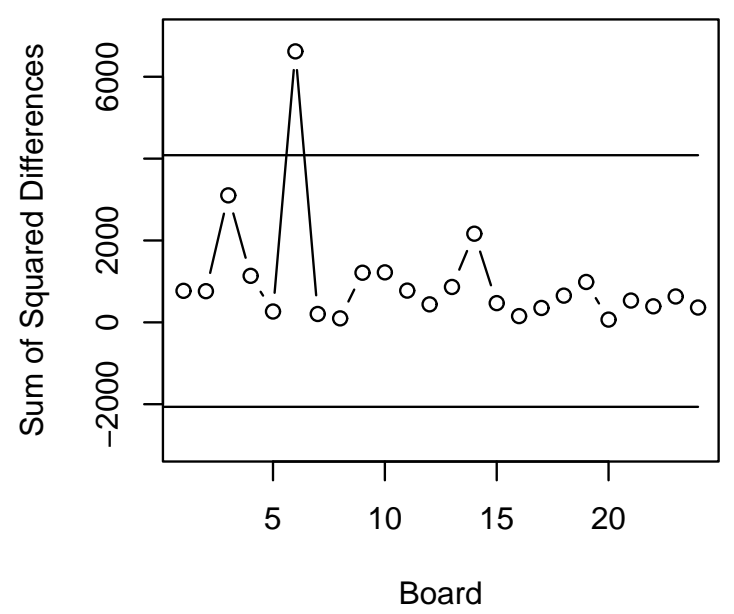

(b)

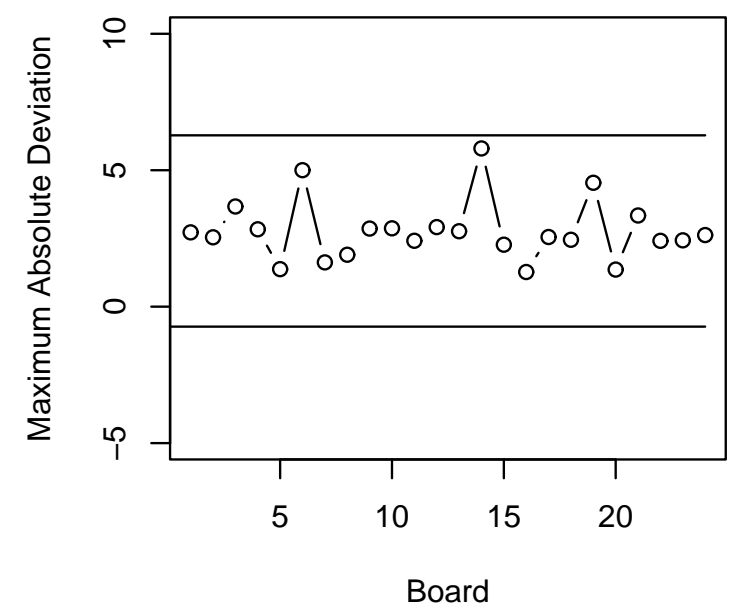

(d)

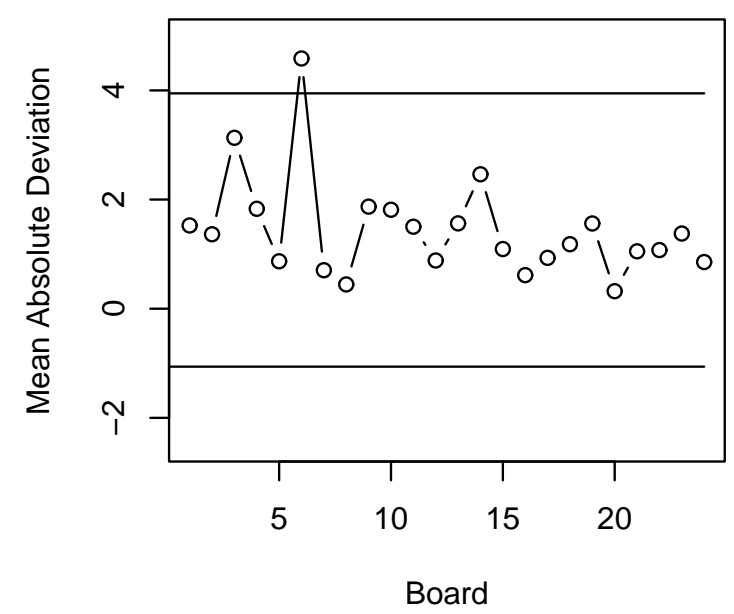

